\title{
Role of histone modification in 12-lipoxygenase-associated p21 gene regulation
}

\author{
YINGCHUN CUI ${ }^{1}$, NIAN LIU ${ }^{2}$, FUZHE MA $^{3}$, WEIXIA SUN ${ }^{3}$, HAO WU $^{3}$, ZHONGGAO XU $^{3}$ and HANG YUAN ${ }^{3}$ \\ ${ }^{1}$ Department of Nephrology, Second Affiliated Hospital of Jilin University; ${ }^{2}$ Center of Urology; ${ }^{3}$ Department of Nephrology, \\ First Affiliated Hospital of Jilin University, Changchun, Jilin 130021, P.R. China
}

Received June 24, 2015; Accepted June 21, 2016

DOI: $10.3892 / \mathrm{mmr} .2016 .5724$

\begin{abstract}
The aim of the present study was to examine the impacts and mechanisms of 12-lipoxygenase (12-LO) and its metabolites on the acetylation and methylation of histone-3-lysine $(\mathrm{H} 3 \mathrm{~K})$ in the p21 gene. Rat mesangial cells (MCs) were selected for use in the present study. A chromatin immunoprecipitation assay, reverse transcription-quantitative polymerase chain reaction analysis and a luciferase assay were used to detect transcriptional activities, the acetylation (Ac) of H3K (H3KAc), p21 promoter methylation (Me) and the transcription regions induced by 12 (S)-hydroxyeicosatetraenoic acid (HETE). The cells were transfected to induce the overexpression of p300 to examine changes in 12 (S)-HETE-associated p21 regulation and epigenetic modifications. 12 (S)-HETE enhanced p21 transcriptional activity and mRNA expression. In the promoter regions, $\mathrm{P} 1$ and $\mathrm{P} 2$, and the $\mathrm{T} 1$ transcription region, 12 (S)-HETE induced significant H3K9 Ac and H3K4 Me1 epigenetic modifications, however, no changes were observed in the $\mathrm{T} 2$ region. By contrast, 12 (S)-HETE treatment markedly prevented H3K9Me3 at the p21 promoter, suggesting that complex Me was involved in 12 (S)-HETE-associated p21 regulation. Furthermore, the overexpression of p300 markedly enhanced basal and 12 (S)-HETE-associated p21 transcriptional regulation in the MCs. 12 (S)-HETE treatment also induced histone acetyltransferase p300 occupancy in the p21 promoter, and reduced the nuclear expression and occupancy of lysine-specific demethylase (LSD1) in the p21 promoter. 12 (S)-HETE induced p300 occupancy, and reduced the nuclear expression and occupancy of LSD1 in the p21 promoter. Therefore, enhanced H3K9Ac and $\mathrm{H} 3 \mathrm{~K} 4 \mathrm{Me} 1$ in the $\mathrm{p} 21$ promoter and transcription regions, and decreased $\mathrm{H} 3 \mathrm{~K} 9 \mathrm{Me} 3$ in the p21 promoter increased the expression of $\mathrm{p} 21$.
\end{abstract}

Correspondence to: Professor Hang Yuan, Department of Nephrology, First Affiliated Hospital of Jilin University, 71 Xinmin Street, Changchun, Jilin 130021, P.R. China

E-mail: cnyuanhang@126.com

Key words: 12 (S)-hydroxyeicosatetraenoic acid, p21, histone, epigenetic modification

\section{Introduction}

Glomerular hypertrophy is a characteristic pathological change of diabetic nephropathy (DN), which predominantly appears as hypertrophy of renal cells and the progressive accumulation of external extracellular matrix. Cellular hypertrophy appears as an increase in cell size, rather than cell number, and its occurrence is closely associated with regulation of the cell cycle (1). In diabetes, cyclin-dependent kinase inhibitors, represented by the p21 protein, are overexpressed, thus the DNA replication is affected and cell proliferation is inhibited (2), which further leads to the occurrence of hypertrophy.

12-lipoxygenase (12-LO) is a type of oxygenase, which is involved in in vivo oxidation reactions of polyunsaturated fatty acids, including arachidonic acid and linoleic acid, and its metabolites may be involved in the occurrence and development of diabetes through oxidative stress and inflammatory responses (3). A previous study confirmed that $12-\mathrm{LO}$ was involved in the pathological changes of diabetic kidney hypertrophy, and was identified as an important factor in DN progression and glomerular fibrosis. Therefore, controlling the expression of 12-LO may assist in reducing the development of renal cell hypertrophy and delay the progression of glomerular sclerosis $(4,5)$. Our preliminary investigations confirmed that the metabolite of 12-LO, 12 (S)-hydroxyeicosatetraenoic acid [12 (S)-HETE], can induce an increase in the protein expression levels of p21 and p27 in mesangial cells, and upregulate the gene expression of p21 through transcription (6). However, the exact regulatory mechanisms remain to be elucidated.

The caudal amino acid residues of histones can affect the density of the DNA double helix through acetylation, methylation, phosphorylation and other modifications, and are thus involved in the transcriptional regulation of genes, known as epigenetic regulation. Previous investigations have confirmed that high blood sugar levels are involved in the occurrence of $\mathrm{DN}$ and other vascular complications by affecting the apparent transcriptional regulation $(7,8)$. In addition, the angiotensin II receptor antagonist, losartan, can affect the chemical modification of histones, indicating the effects of reducing proteinuria and inhibiting glomerular sclerosis, and suggesting that the chemical modification of histones may be a novel mechanism and target in the intervention of vascular complications in diabetes $(9,10)$. Our preliminary results confirmed that the acetylation of histones involved the regulation by transforming 
growth factor (TGF- $\beta 1)$ on the expression of the pro-fibrotic gene, PAI-1 $(11,12)$. As the downstream regulatory factor of the TGF- $\beta 1$ pathway, $12-\mathrm{LO}$ and the TGF- $\beta 1$ pathway may mutually activate each other, thus jointly mediating the high expression levels of PAI-1, collagen and fibronectin under high glucose conditions (6). However, whether 12 (S)-HETE is involved in the gene expression of p21 through epigenetic regulation (chemical modification of histones) has not been reported. For the first time, to the best of our knowledge, the present study used chromatin co-immunoprecipitation technology to examine the effects and mechanisms of 12-LO, and its metabolites, on the chemical modification (acetylation and methylation) of histones in the p21 gene. Thus, the present study demonstrated the epigenetic modification mechanism associated with the overexpression of cyclin-dependent kinase inhibitor p21 under diabetic conditions, and provides a noveltheoretical basis and intervention targets to delay diabetic glomeruli hypertrophy.

\section{Materials and methods}

Cell culture. The primary cultured rat MCs were isolated from Sprague-Dawley rats and cultured as previously described (13) and were seeded into culture dishes with RPMI 1640 medium (Gibco; Thermo Fisher Scientific, Inc., Waltham, MA, USA) and cultured at $37^{\circ} \mathrm{C}$ in $5 \% \mathrm{CO}_{2}$. When the cells fused and covered $80 \%$ of the bottom of the petri dish, the serum-free medium was replaced for synchronic culture for another $24 \mathrm{~h}$ for the subsequent experiments. The present study was approved by the ethics committee of the First Affiliated Hospital of Jilin University (Changchun, China).

Reverse transcription-quantitative polymerase chain reaction (RT-qPCR) analysis. An RNA STAT60 kit (AMS Biotechnology Europe Ltd., Abingdon, UK) was used to extract the total mRNA from the MCs, and a TaqMan PreAmp Master mix (Applied Biosystems; Thermo Fisher Scientific, Inc.) was then used to reverse transcribe $1.5 \mu \mathrm{g}$ mRNA into cDNA, the final product was diluted 10-fold and used as the template for PCR amplification. An ABI-7500 real-time quantitative PCR instrument (Thermo Fisher Scientific, Inc.) was used, together with the SYBR Green PCR Master Mix kit (Thermo Fisher Scientific, Inc.). The reaction volume, of $20 \mu 1$, comprised $10 \mu 1$ SYBR Green mix, $1 \mu 1$ of the positive and negative primer strands (50 pmol), respectively, $5 \mu \mathrm{l}$ cDNA template and $3 \mu \mathrm{l}$ DEPC water. The thermocycling conditions were as follows: $50^{\circ} \mathrm{C}$ for $2 \mathrm{~min}$ and $95^{\circ} \mathrm{C}$ for $10 \mathrm{~min} ; 40$ cycles of $95^{\circ} \mathrm{C}$ for $15 \mathrm{sec}, 60^{\circ} \mathrm{C}$ for $1 \mathrm{~min}$; followed by melt curve analysis at $95^{\circ} \mathrm{C}$ for $15 \mathrm{sec}, 60^{\circ} \mathrm{C}$ for $1 \mathrm{~min}, 95^{\circ} \mathrm{C}$ for $30 \mathrm{sec}$ and $60^{\circ} \mathrm{C}$ for $1 \mathrm{~min}$. Primers are presented in Table I. $\beta$-actin was selected as the internal control, and the $2^{-\Delta \Delta \mathrm{Cq}}$ method was used for the analysis as described previously (13).

Western blot analysis. Cells were lysed in 1.5X sodium dodecyl sulfate (SDS) buffer and protein concentration was estimated using the Lowry method. Equal quantities of the samples $(50 \mu \mathrm{g})$ were separated by electrophoresis on a $10 \%$ SDS gel and transferred onto a nylon membrane. Following treatment with closure fluid, rabbit antibodies against p21 (1:500; Santa Cruz Biotechnology, Inc., Dallas, TX, USA; cat. no. sc-756) lysine-specific demethylase (LSD1; 1:1,000; Cell Signaling Technology, Inc., Danvers, MA, USA; cat. no. 2139) and mouse antibodies against p300 (1:500; EMD Millipore, Billerica, MA, USA; cat. no. 05-257) were added for overnight incubation at $4^{\circ} \mathrm{C}$, and the membrane was rinsed. Horseradish peroxidase-labeled goat anti-rabbit (1:1,000; cat. no. ER48616) and goat anti-mouse (1:1,000; cat. no. ER48627) secondary antibodies (Sigma-Aldrich, St. Louis, MO, USA) were then added for hybridization at room temperature for $1 \mathrm{~h}$, following which the membrane was washed and ECL reagent was added for staining and developing images as described previously (6). Images of the blots were analyzed using Quantity One software (Bio-Rad Laboratories, Inc., Hercules, CA, USA). The nucleoprotein extraction method was performed, according to the protocol of the Nucleoprotein Extraction kit (Thermo Fisher Scientific, Inc.).

Chromatin immunoprecipitation (ChIP assay). Following stimulation with 12 (S)-HETE, the MCs were fixed in $1 \%$ formalin, sonicated and pretreated with protein A/G-immunomagnetic beads (Invitrogen; Thermo Fisher Scientific, Inc.), followed by the addition of specific histone-3-lysine (H3K)9Ac (1:50; EMD Millipore; cat. no. 06-599), H3K4Me1 (1:50; Abcam, Cambridge, MA, USA; cat. no. ab8895), H3K9Me3 (1:50; Abcam; cat. no. ab8898), p300 (1:50) and LSD1 (1:50) antibodies or the internal reference antibody (mouse and rabbit IgG, cat. nos. 12-371 and 12-370, respectively), obtained from EMD Millipore for overnight incubation (EZ ChIP kit; EMD Millipore). The precipitated and separated DNA fragments were then used as the template for qPCR amplification using the ABI-7500 real-time quantitative PCR instrument. The $2^{-\Delta \Delta \mathrm{Cq}}$ method was used to analyze the results. The primer sequences and the amplification regions are shown in Table I and Fig. 1.

Construction and amplification of plasmids. According to the nucleotide sequence of the rat $\mathrm{p} 21$ gene promoter region (GenBank), Invitrogen primer design software (Thermo Fisher Scientific, Inc.) was used to amplify the nucleotide sequence of the p21 genome between DNA -4,542 and +113; the amplified DNA fragment was then inserted using the enzyme-linked method, into a pGL3 vector (Promega Corporation, Madison, WI, USA), which contained the luciferase reporter gene, to synthesize pGL3-p21 according to our previous study (11). The recombinant was then transformed into competent Escherichia coli (donated by Professor Wang Fang, Department of Microbiology, Bethune Medical School of Jilin University, Changchun, China), which were extracted using resistance screening for identification and future use. The p300 expression plasmid was provided by Dr B. Forman (Beckman Research Center, Duarte, CA, USA).

Cell transfection and analysis of luciferase activity. FuGene 6 (Roche, Basel, Switzerland) was used as the transfection reagent for the transfection procedures. The cells in the control group were transfected with a plasmid containing the green fluorescent protein sequence, according to the protocol of the Nucleofecter ${ }^{\mathrm{TM}}$ kit (Lonza Group, Basel, Switzerland). A luciferase activity detection kit (Promega Corporation) and TD-20/20 luciferase activity analyzer (Promega Corporation) 
Table I. Primer sequences.

\begin{tabular}{lll}
\hline Primer & \multicolumn{1}{c}{ Forward (5'-3') } & \multicolumn{1}{c}{ Reverse (5'-3') } \\
\hline ChIP assay & & \\
p21 P1 (R) & AGTGGATTCAAACATATGAGCCACT & CCCTCCATCCCCCAAGGCCCTG \\
p21 P2 (R) & GTTCAGCCCTGGAACCGAAG & GTACCAAACACCCTTCACCTGGTAC \\
p21 T1 (R) & GGCGCTCAGCCATTCAGTAT & CGTGACCAGGGATAGGCTGTCACG \\
p21 T2 (R) & CGGCCAGTGAGCAGTTGAGCcG & CCCTCCAGTGGCGTCTCAGT \\
cDNA & & \\
p21 (R) & GTGGCCTTGTCGCTGTCTTG & CGATTCTTGCAGAAGACCAATCG \\
$\beta$-actin (R) & CCCTGTATGCCTCTGGTCGT & CGGACGCAGCTCAGTAACAGTCCG \\
\hline
\end{tabular}

ChIP, chromatin immunoprecipitation; R, rat; P1, promoter 1; P2, promoter 2; T1, transcription region 1; T, transcription region 2.

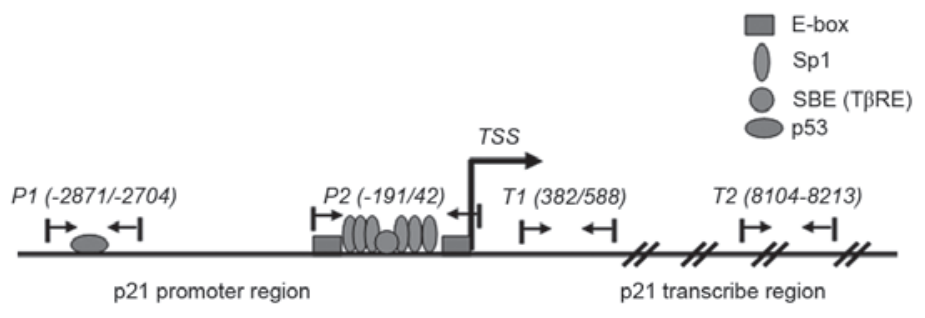

Figure 1. Locations of the p21 promoter and transcription primers for the ChIP-qPCR assay. The locations of the p21 promoter and transcription primers for ChIP-qPCR are shown. P, promoter primer; T, transcription primer; SBE, Smad binding element; Sp1, Sp1 binding sites; p53: p53 binding sites; TSS, transcription start site; ChIP-qPCR. chromatin immunoprecipitation-quantitative polymerase chain reaction.

were used to analyze the luciferase activities of pGL-3 and pRL-TK, according to the kit protocol.

Statistical analysis. Data were expressed as the mean \pm standard error of the mean from multiple experiments. GraphPad Prism 5.0 software (GraphPad Software, Inc., La Jolla, CA, USA) was used for data analysis. Intergroup comparison was performed using paired Student's t-test; multigroup comparisons were performed using analysis of variance with Dunnett's post-hoc tests. $\mathrm{P}<0.05$ was considered to indicate a statistically significant difference.

\section{Results}

Expression and transcriptional activity of the p21 gene. Following synchronic culture in serum-free medium for $24 \mathrm{~h}$, 12 (S)-HETE $\left(10^{-6}, 10^{-7}\right.$ and $\left.10^{-8}\right)$ was added to the MCs for 2,4 and $8 \mathrm{~h}$, the cells were collected to extract mRNA for RT-qPCR analysis. The results (Fig. 2A) showed that 12 (S)-HETE promoted the intracellular mRNA expression of $\mathrm{p} 21$, and the effects stimulated by the $10^{-7} \mathrm{M}$ concentration were the most marked $(\mathrm{P}<0.01)$. Within the stimulation period $(2-8 \mathrm{~h})$, the mRNA expression of p21 was increased by 2.5-2.8, compared with the control group. In order to determine whether the regulation was through the transcriptional pathway, when the MCs grew and covered $70-80 \%$ of the bottom of the petri dish, the pGL3-p21 plasmid was transfected into the cells using Fugene 6 . After $6 \mathrm{~h}$, the serum-free medium was replaced prior to incubation for another $24 \mathrm{~h}$, following which 12 (S)-HETE $\left(10^{-7} \mathrm{M}\right)$ was added for $0.5-8 \mathrm{~h}$ stimulation. The cells were then collected for the detection of luciferase activity. As shown in Fig. 2B, following stimulation with 12 (S)-HETE for $0.5 \mathrm{~h}$, the transcriptional activity of p21 began to increase, which continued until $8 \mathrm{~h}(\mathrm{P}<0.001)$. This further confirmed that 12 (S)-HETE promoted the gene expression of p21 via the transcriptional pathway.

Modification of the 221 gene promoter and transcription regions of $\mathrm{H} 3 \mathrm{~K} 9 \mathrm{Ac}, \mathrm{H} 3 \mathrm{~K} 4 \mathrm{Me} \mathrm{I}$ and $\mathrm{H} 3 \mathrm{~K} 9 \mathrm{Me} 3$. In order to confirm whether the chemical modification of histones, via acetylation and methylation, was involved in the transcriptional regulation of 12 (S)-HETE towards the p21 gene, a ChIP assay was performed to observe the changes in the levels of chemical modification of $\mathrm{H} 3 \mathrm{~K}$ in the p21 gene promoter $(\mathrm{P})$ and transcription $(\mathrm{T})$ regions. As shown in Fig. 3, the results showed that 12 (S)-HETE significantly increased the level of H3K9Ac in P1 and P2 of the p21 gene $(\mathrm{P}<0.001)$. The level of H3K9Ac was also significantly increased in $\mathrm{T} 1(\mathrm{P}<0.01)$, whereas the level of H3K9Ac in T2 (8,104-8,213 bp), far from the transcription start site (TSS), did not alter significantly. Compared with acetylation, the mechanisms underlying the effects of methylated histones in the regulation of gene expression were relatively complex. It was confirmed that methylation at the H3K4 site was involved in the transcriptional activation, whereas the methylation at $\mathrm{H} 3 \mathrm{~K} 9$ suppressed transcriptional activation. As shown in Fig. 4A, 12 (S)-HETE increased the modification of $\mathrm{H} 3 \mathrm{~K} 4 \mathrm{Me} 1$ in $\mathrm{P} 2$ and T1, close to TSS, and in P1, however, no significant difference was found in T2. It was found that the stimulation of 12 (S)-HETE in P1 and P2 significantly 
A

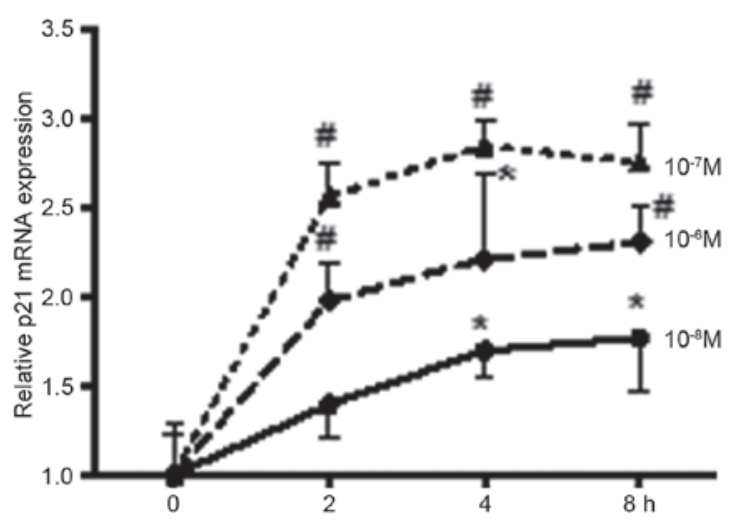

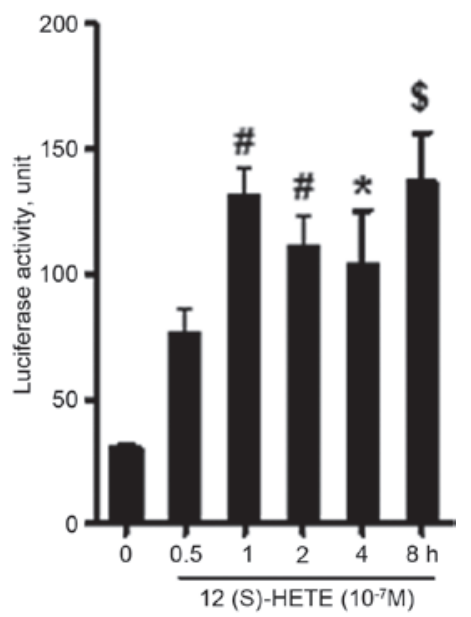

Figure 2. 12 (S)-HETE induces the mRNA expression and transcriptional activity of p21. (A) Serum depleted rat MCs were treated with different concentrations of $12(\mathrm{~S})$-HETE $\left(10^{-6}-10^{-8} \mathrm{M}\right)$ at various time points $(2-8 \mathrm{~h})$, and the mRNA level of p21 was analyzed using reverse transcription-quantitative polymerase chain reaction analysis. (B) MCs were transfected with the p21 promoter-luciferase construct, and then treated without or with 12 (S)-HETE (10 $\left.{ }^{-7} \mathrm{M}\right)$ for the indicated durations. Luciferase activities in the cell lysates were determined. Values are presented as the mean \pm standard error of the mean $(n=3)$. $P<0.05$, ${ }^{\#} \mathrm{P}<0.01$ and ${ }^{\$} \mathrm{P}<0.001$, vs. corresponding untreated control. MCs, mesangial cells; 12 (S)-HETE, 12 (S)-hydroxyeicosatetraenoic acid.

A

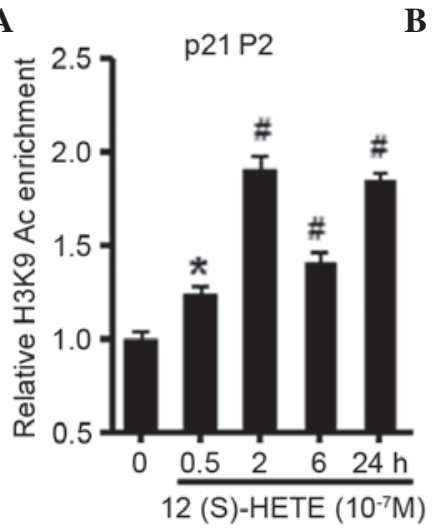

B

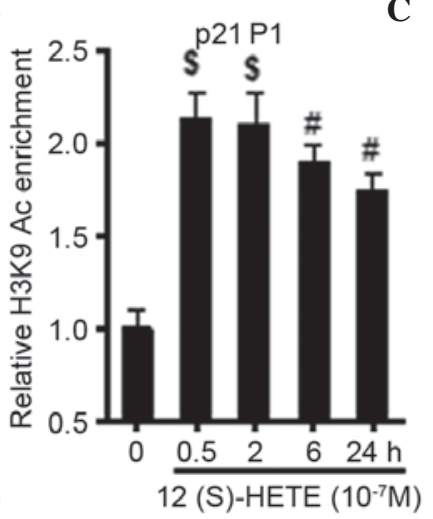

C

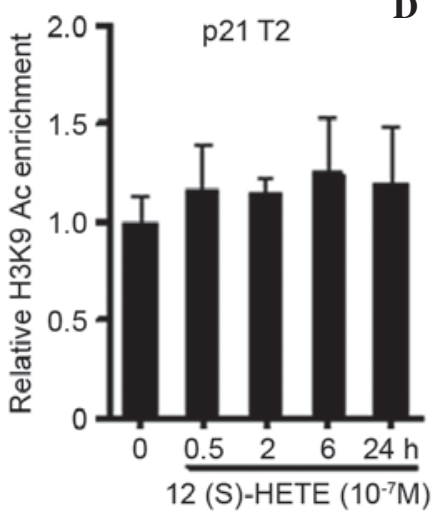

D

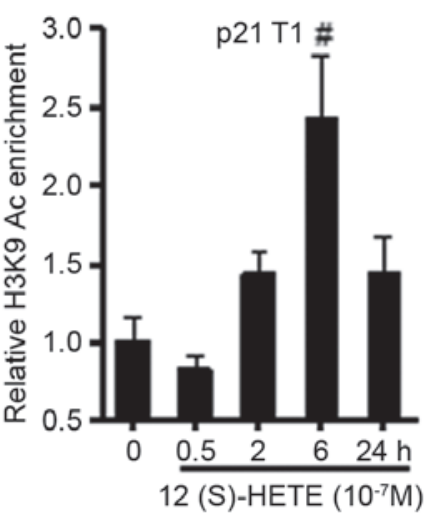

Figure 3. 12 (S)-HETE treatment induces H3K9Ac enrichment at p21 promoter and transcription regions. Quiescent rat MCs were treated with 12 (S)-HETE (10-7 M) for the indicated time course. Chromatin immunoprecipitation assays were performed with H3K9Ac antibody. Immunoprecipitated DNA and input DNA were subjected to qPCR analysis with primers specific for the indicated gene (A and B) promoter and (C and D) transcription regions to measure enrichment levels. The qPCR data were analyzed using the $2^{-\triangle \Delta C q}$ method, and the results normalized to input DNA are expressed as the fold change over the respective untreated control cells. Values are presented as the mean \pm standard error of the mean $(\mathrm{n}=3)$. ${ }^{*} \mathrm{P}<0.05$, ${ }^{*} \mathrm{P}<0.01$ and ${ }^{\$} \mathrm{P}<0.001$, vs. corresponding untreated control. MCs, mesangial cells; H3K, histone-3-lysine; 12 (S)-HETE, 12 (S)-hydroxyeicosatetraenoic acid; qPCR, quantitative polymerase chain reaction.

reduced the modification of $\mathrm{H} 3 \mathrm{~K} 9 \mathrm{Me} 3$ ( $\mathrm{P}<0.01$; Fig. $4 \mathrm{~B})$, whereas no significant difference was found in the transcription region. These results suggested that 12 (S)-HETE increased the modification of H3K9Ac and H3K4Me1 in p21, and reduced the modification of $\mathrm{H} 3 \mathrm{~K} 9 \mathrm{Me} 3$ in the promoter region, thus promoting the transcriptional expression of $\mathrm{p} 21$.

Effect of the level of histoneacetyl transferase (HAT; p300) on the transcriptional regulation of 12 (S)-HETE on the expression of $p 21$. As shown in Fig. 5A, the results showed that, following transfection with the p300 expression vector, the intracellular protein level of p300 was significantly increased, and the protein level of p21 was also increased. In the group of cells expressing a high level of P300, 2 h stimulation with $12(\mathrm{~S})$-HETE $\left(10^{-7} \mathrm{M}\right)$ significantly increased the transcriptional activity of the p21 promoter region (Fig. 5B) and its mRNA level (Fig. 5C), compared with the control group and 12 (S)-HETE stimulation only group, suggesting that increasing the intracellular level of HAT (p300) significantly promotes the 12 (S)-HETE-induced transcriptional expression of p21. The results of the western blot analysis (Fig. 5D) further confirmed that the increased expression of HAT (p300) increased the intracellular protein expression of p21 and the promoting effect of 12 (S)-HETE on the expression of $\mathrm{p} 21$.

Level of LSD1 in the nuclei and its binding in the promoter region of the p21 gene. To further investigate the mechanism of 12 (S)-HETE-induced chemical modification of histones in the p21 gene, the present study examined the binding of HAT (p300) and demethylase LSD1 in the p21 promoter region following stimulation with 12 (S)-HETE $\left(10^{-7} \mathrm{M}\right)$. The ChIP assay results (Fig. 6A) showed that 12 (S)-HETE promoted the binding of p300 in $\mathrm{P} 2(\mathrm{P}<0.01)$, whereas no significant changes were observed in the $\mathrm{P} 1$ or transcription regions, suggesting that the modification of H3K9Ac in $\mathrm{P} 2$ was associated with the increased binding of p300 in this area. As shown in Fig. 6B, 

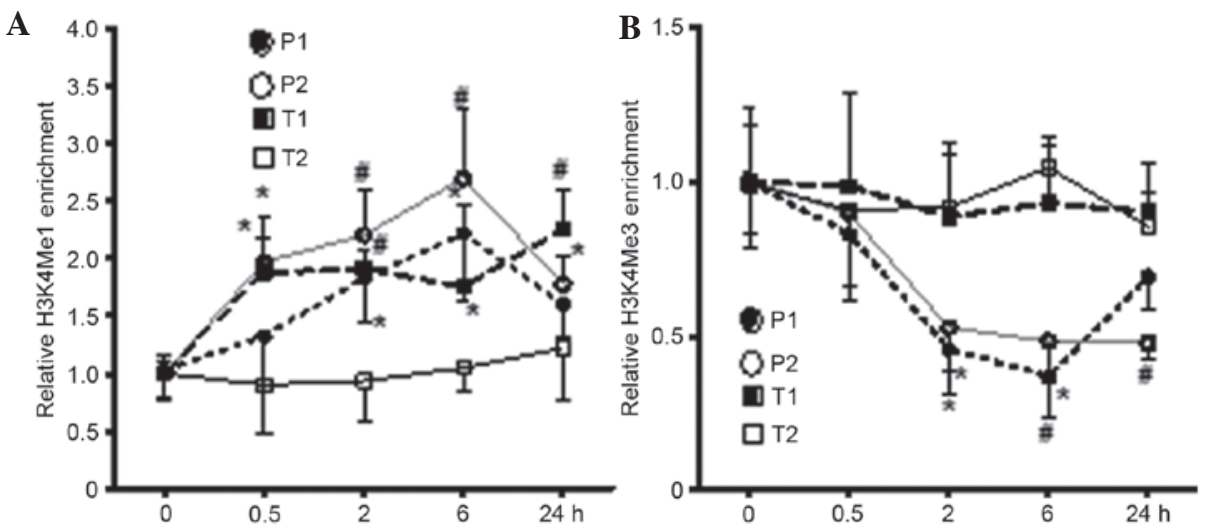

Figure 4. 12 (S)-HETE induces H3K4Me1 and H3K9Me3 epigenetic modifications in the p21 promoter and transcription regions. Quiescent rat MCs were treated with 12 (S)-HETE (10-7 M) for the indicated time course. (A) H3K4Me1 and (B) H3K9Me3 enrichment at indicated promoter and transcription regions were detected using chromatin immunoprecipitation assays. Data from quantitative-polymerase chain reaction analysis were analyzed using the $2^{-\Delta \Delta C q}$ method, and results normalized to input DNA were expressed as the fold change over the respective untreated control cells. Values are presented as the mean \pm standard error of the mean $(\mathrm{n}=3) .{ }^{*} \mathrm{P}<0.05,{ }^{*} \mathrm{P}<0.01$ vs. corresponding untreated control. MCs, mesangial cells; 12 (S)-HETE, 12 (S)-hydroxyeicosatetraenoic acid; H3K, histone-3-lysine; P1, protomer 1, P2, promoter 2; T1, transcription region 1; T2, transcription region 2.

12 (S)-HETE significantly inhibited the binding of LSD1 in $\mathrm{P} 1$ and $\mathrm{P} 2(\mathrm{P}<0.01)$, whereas no significant difference in the transcription region was observed, compared with the control group, suggesting that 12 (S)-HETE inhibited the binding of LSD1 in the promoter region, thereby inducing the modification of H3K4Me1 in P2. Further investigation (Fig. 6C) showed that $12(\mathrm{~S})$-HETE $\left(10^{-7} \mathrm{M}\right)$ stimulated the reduction in the protein level of LSD1 in the nuclei, suggesting that 12 (S)-HETE induced the modification of H3K4Me1 in p21 through inhibiting the expression of LSD1 in the nuclei, thus contributing to the expression of $\mathrm{p} 21$.

\section{Discussion}

DM is a chronic metabolic disease, which is affected by multiple and complex factors, and the occurrence of which can be caused by alterations in genetic and environmental factors. Studies have confirmed that, prior to the diagnosis of DM, patients present with abnormal glucose tolerance and the body has already formed metabolic memory, presenting potential hidden risks for the occurrence of DM and its complications (14-16), however, it also indicates that abnormal epigenetic modifications may be involved in the pathogenesis of DM and its complications $(17,18)$.

The acetylation of histones occurs predominantly under the coordination of HATs and histone deacetylases (HDACs). HATs can catalyze the acetylation of histones, resulting in a loose chromatin structure and the promotion of gene transcription, whereas HDACs induce the deacetylation of histones, resulting in the condensation of chromatin and inhibition of gene transcription. Compared with the acetylation of histones, the methylation of histones is more stable and lasting, according to the methylation patterns, sites and modified amino acid residues. The methylation of histones can lead to transcriptional activation or inhibition, for example H3K9Me can inhibit transcription, whereas $\mathrm{H} 3 \mathrm{~K} 4 \mathrm{Me}$ can lead to transcriptional activation (19). Different types of chemical modification at the same site of histones can affect each other, thus co-acting on the regulation of gene expression. H3K9Me3 is important in gene silencing, which can inhibit transcriptional activity by increasing the binding of HDAC on the DNA chain (20).

TGF- $\beta 1$ is an important pathogenic factor in the pathogenesis of DN, which can induce the modifications of H3K9Ac and H3K4Me, thus affecting the transcription level of plasminogen activator inhibitor- 1 and other genes, and inducing the occurrence of renal fibrosis $(11,21)$. Our previous study found that 12 (S)-HETE increases the protein expression levels of cyclin inhibitor p21 and p27, and is involved in the occurrence and development of DN (6). In the present study, the ChIP assay revealed that 12 (S)-HETE induced the modification of H3K9Ac in the p21 gene promoter region and the TSS-adjacent transcription region. 12 (S)-HETE also stimulated and increased the modification of $\mathrm{H} 3 \mathrm{~K} 4 \mathrm{Me} 1$ in TSS-adjacent P2 and $\mathrm{T} 1$, and reduced the level of $\mathrm{H} 3 \mathrm{~K} 9 \mathrm{Me} 3$ modification in the promoter region.

In investigating the mechanism of the chemical modification of histones in regulating the gene expression of $\mathrm{p} 21$, the present study found that the increased expression of HAT (p300) significantly increased the promoting effect of 12 (S)-HETE on the gene expression of $\mathrm{p} 21$, and this further confirmed the role of acetylation in the transcriptional regulation of the p21 gene. The ChIP assay showed that 12 (S)-HETE induced the binding of p300 in P2, suggesting that the 12 (S)-HETE P2-induced modification of H3K9Ac was associated with the binding of the p300 protein in $\mathrm{p} 21$. The acetylation in P1 may be due to fact that this region contains the binding sites of $\mathrm{p} 53$, and the p53 protein can induce modifications in the binding region through acetylation or binding with other HATs (22-24).

The methylation of histone H3K4 is usually associated with active gene expression. H3K4 can be monomethylated, dimethylated or trimethylated on lysine residues by specific histone H3K4 methyltransferases, including SET7/9 or mixed-lineage leukemia (20). The previous identification of LSD1, which specifically removes $\mathrm{H} 3 \mathrm{~K} 4 \mathrm{me} 1$ and $\mathrm{H} 3 \mathrm{~K} 4 \mathrm{me} 2$, demonstrated the dynamic nature of $\mathrm{H} 3 \mathrm{~K}$ methylation $(25,26)$. In the present study, it was found that 12 (S)-HETE reduced the protein level of LSD1 within nuclei and inhibited its binding in the promoter region of the p21 gene, suggesting that it may be the primary 
B

A
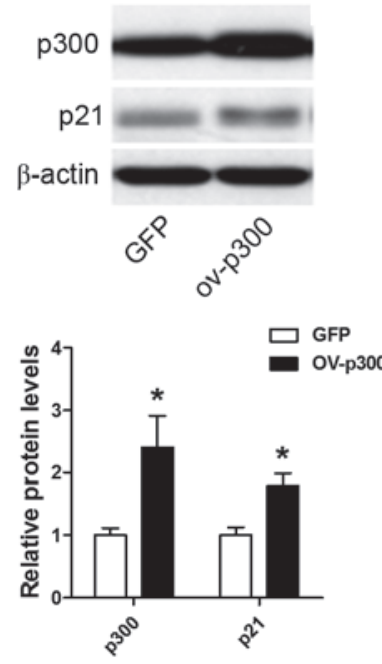

C

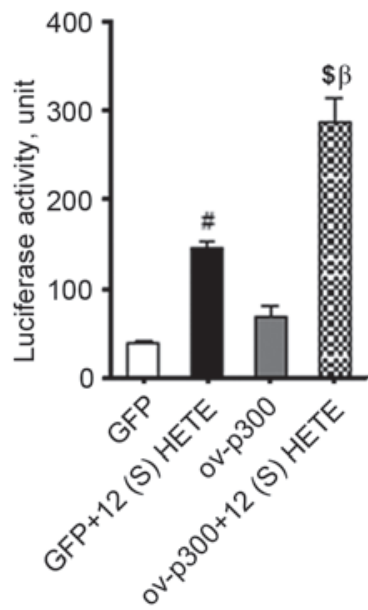

D
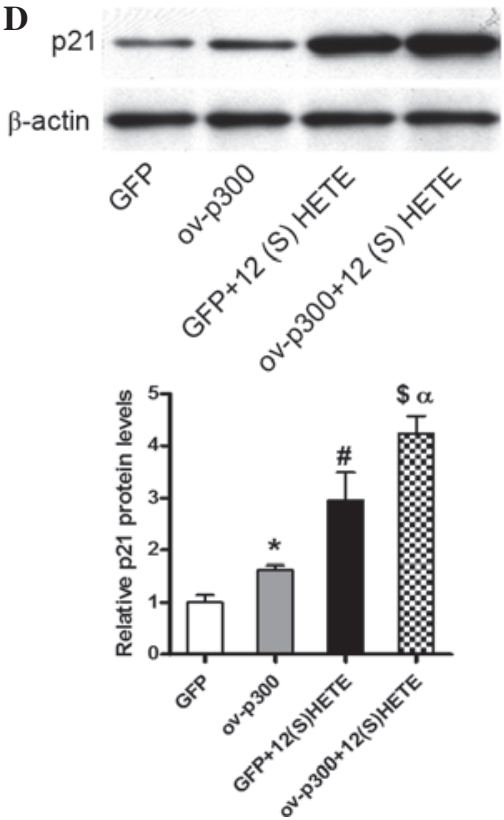

Figure 5. Overexpression of p300 enhances basal and 12 (S)-HETE-associated gene expression and transcriptional activity of p21 (A) Rat MCs were transiently transfected with either a p300 expression vector or a control enhanced GFP expression vector, followed by western blot analysis. Bar graph demonstrates quantification of immunoblotting, a significant increase in p300 and p21 protein expression levels in p300-overexpressing cells relative to GFP was observed. (B) Rat MCs were co-transfected with the p300 expression vector or GFP expression vector, combined with a p21 promoter-luciferase construct, and then treated without or with $12(\mathrm{~S})$-HETE $\left(10^{-7} \mathrm{M}\right)$ for $2 \mathrm{~h}$. The luciferase activity in the cell lysates were determined. Rat MCs were co-transfected with the p300 expression vector or a GFP expression vector, and then treated with $12(\mathrm{~S})$-HETE $\left(10^{-7} \mathrm{M}\right)$ for $2 \mathrm{~h}$. Total RNA and proteins were extracted for $(\mathrm{C})$ quantitative polymerase chain reaction and (D) western blot analyses to determine the changes in the expression of $\mathrm{p} 21$. Bar graph represent quantification of immunoblotting. Values are presented as the mean \pm standard error of the mean $(n=3) .{ }^{*} \mathrm{P}<0.05,{ }^{\#} \mathrm{P}<0.01$ and ${ }^{\$} \mathrm{P}<0.001$, vs. GFP; ${ }^{a} \mathrm{P}<0.05$ and ${ }^{\mathrm{B}} \mathrm{P}<0.01$, vs. GFP+12 (S)-HETE MCs, mesangial cells; 12 (S)-HETE, 12 (S)-hydroxyeicosatetraenoic acid; GFP, green fluorescence protein.

A

$\mathbf{B}$

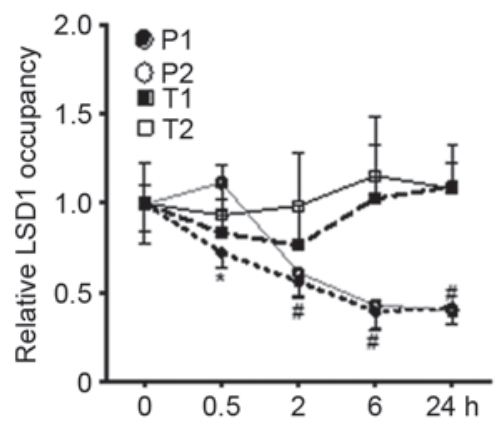

C

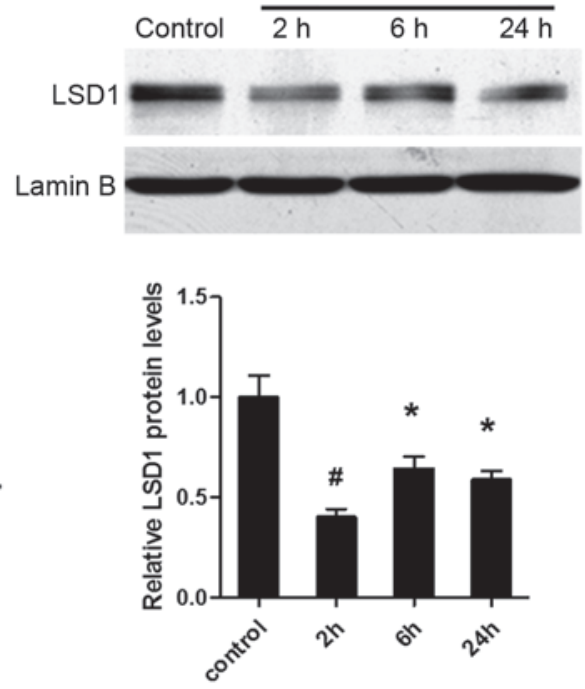

Figure 6. Effect of 12 (S)-HETE on the levels of p300 and LSD1 in the p21 promoter and transcription regions. Quiescent rat MCs were treated with 12 (S)-HETE (10-7 M) for the indicated time course. The relative occupancy of (A) p300 and (B) LSD1 at indicated promoter and transcription regions of p21 were detected using ChIP assays. Quantitative polymerase chain reaction data were analyzed using the $2^{-\triangle A C q}$ method and results, normalized to input DNA, are expressed as the fold change over the respective untreated control cells. Values are presented as the means \pm standard error of the mean ( $n=3$ ). (C) Quiescent rat MCs were treated with 12 (S)-HETE (10-7 M) for the indicated time course, followed by western blot analysis of nuclear protein lysates from the untreated control and 12 (S)-HETE-treated MCs using LSD1 and Lamin B antibodies. Bar graph quantification showing a significant decrease in LSD1 protein levels by 12 (S)-HETE treatment. " $\mathrm{P}<0.05,{ }^{~}{ }^{\mathrm{P}}<0.01$ vs. control cells. MCs, mesangial cells; H3K, histone-3-lysine; 12 (S)-HETE, 12 (S)-hydroxyeicosatetraenoic acid; LSD1, lysine-specific demethylase; ChIP,. chromatin immunoprecipitation; P1, protomer region 1, P2, promoter region 2; T1, transcption region 1; T2, transcription region 2 .

cause of the H3K4Me1 modification in this region. Previous studies have shown that the H3K4Me modifications of histones can competitively inhibit the binding of HDAC and DNA, thus promoting H3K9Ac and suppressing the occurrence of 
H3K9Me (27,28). The present study showed that, in addition to 12 (S)-HETE-induced H3K4Me1 modification in the p21 gene promoter region, the gene silencing-associated H3K9Me3 modification was significantly decreased simultaneously, indicating that the transcriptional regulation of 12 (S)-HETE towards the p21 gene was realized through comprehensive chemical modification of histones. Due to the complexity of the methylation regulation of histones and the numerous factors affecting this, the specific pathogenesis requires further investigation.

In conclusion, the present study demonstrated the following: The acetylation and methylation of histones were closely associated with the 12 (S)-HETE-induced transcriptional regulation towards the gene expression of p21; 12 (S)-HETE inhibited the expression of LSD1 and its binding in the $\mathrm{p} 21$ gene promoter region, thus inducing the modification of $\mathrm{H} 3 \mathrm{~K} 4 \mathrm{Me} 1$ in the $\mathrm{p} 21$ gene promoter region. Increasing the binding of p300 in the p21 gene promoter region induced the modification of H3K9Ac and 12 (S)-HETE stimulation inhibited the level of H3K9Me3 in the p21 promoter region. These changes resulted in the p21 gene promoter and peri-TSS chromatin being in the relaxed transcription activation state, thus transcriptional activity was increased and the expression of $\mathrm{p} 21$ gene was promoted.

In conclusion, associated epigenetic changes, including increased histone $\mathrm{H} 3 \mathrm{~K} 9 \mathrm{Ac}$ and $\mathrm{H} 3 \mathrm{~K} 4 \mathrm{Me} 1$, and reduced H3K9Me3 may involved in 12(S)-HETE mediated p21 gene regulation, and thus, be important in the pathogenesis of renal hypertrophy in diabetes.

\section{Acknowledgements}

This study was supported by the Youth Foundation of national natural Science foundation of China (grant no. 81000300); The National Natural Science Foundation of China (grant nos. 81370830, 81170669, 81070578 and 81270809), the Jilin Provincial Department of Science and Technology (grant no. 20130521006JH), the Doctor Foundation, Ministry of Education of P.R. China (grant no. 2010006120024) and the Item of Bethune Project B, Jilin University (grant no, 2012226).

\section{References}

1. Hostetter TH: Progression of renal disease and renal hypertrophy. Annu Rev Physiol 57: 263-278, 1995.

2. Al-Douahji M, Brugarolas J, Brown PA, Stehman-Breen CO, Alpers CE and Shankland SJ: The cyclin kinase inhibitor p21WAF/CIP1 is required for glomerular hypertrophy in experimental diabetic nephropathy. Kidney Int 56: 1691-1699, 1999.

3. Bleich D, Chen S, Zipser B, Sun D, Funk CD and Nadler JL: Resistance to the type 1 diabetes induction in 12-lipoxygenase knockout mice. J Clin Invest 103: 1431-1436, 1999.

4. Yuan H, Lanting L, Xu ZG, Li SL, Swiderski P, Putta S, Jonnalagadda M, Kato M and Natarajan R: Effects of cholesterol tagged small interfering RNA targeting 12/15-lipoxygenase on parameters of diabetic nephropathy in mouse model of type 1 diabetes. Am J Physiol Renal Physiol 295: F605-F617, 2008.

5. Ma J, Natarajan R, LaPage J, Lanting L, Kim N, Becerra D, Clemmons B, Nast CC, Surya Prakash GK, Mandal M and Adler SG: 12/15-lipoxygenase inhibitors in diabetic nephropathy in the rat. Prostaglandins Leukot Essent Fatty Acids 72: 13-20, 2005.
6. Kim YS, Xu ZG, Reddy MA, Li SL, Lanting L, Sharma K, Adler SG and Natarajan R: Novel interactions between TGF- $\{$ beta\} 1 actions and the 12/15-lipoxygenase pathway in mesangial cells. J Am Soc Nephrol 16: 352-362, 2005.

7. Kato $M$ and Natarajan R: Diabetic nephropathy-emerging epigenetic mechanisms. Nat Rev Nephrol 10: 517-530, 2014.

8. Reddy MA, Tak Park J and Natarajan R: Epigenetic modifications in the pathogenesis of diabetic nephropathy. Semin Nephrol 33: 341-353, 2013.

9. Reddy MA, Sumanth P, Lanting L, Yuan H, Wang M, Mar D, Alpers CE, Bomsztyk K and Natarajan R: Losartan reverses permissive epigenetic changes in renal glomeruli of diabetic $\mathrm{db} / \mathrm{db}$ mice. Kidney Int 85: 362-373, 2014

10. Natarajan R: Drugs targeting epigenetic histone acetylation in vascular smooth muscle cells for restenosis and atherosclerosis. Arterioscler Thromb Vasc Biol 31: 725-727, 2011.

11. Yuan H, Reddy MA, Sun G, Lanting L, Wang M, Kato M and Natarajan R: Involvement of p300/CBP and epigenetic histone acetylation in TGF- $\beta 1$-mediated gene transcription in mesangial cells. Am J Physiol Renal Physiol 304: F601-F613, 2013.

12. Kato M, Dang V, Wang M, Park JT, Deshpande S, Kadam S, Mardiros A, Zhan Y, Oettgen P, Putta S, et al: TGF- $\beta$ induces acetylation of chromatin and of Ets-1 to alleviate repression of miR-192 in diabetic nephropathy. Sci Signal 6: ra43, 2013.

13. Sun G, Reddy MA, Yuan H, Lanting L, Kato M and Natarajan R: Epigenetic histone methylation modulates fibrotic gene expression. J Am Soc Nephrol 21: 2069-2080, 2010.

14. Villeneuve LM and Natarajan R: The role of epigenetics in the pathology of diabetic complications. Am J Physiol Renal Physiol 299: F14-F25, 2010.

15. Reddy MA, Zhang E and Natarajan R: Epigenetic mechanisms in diabetic complications and metabolic memory. Diabetologia 58: 443-455, 2015 .

16. Miao F, Chen Z, Genuth S, Paterson A, Zhang L, Wu X, Li SM, Cleary P, Riggs A, Harlan DM, et al: Evaluating the role of epigenetic histone modifications in the metabolic memory of type 1 diabetes. Diabetes 63: 1748-1762, 2014.

17. Ling C and Groop L: Epigenetics: A molecular link between environmental factors and type 2 diabetes. Diabetes 58: 2718-2725, 2009.

18. Pirola L, Balcerczyk A, Okabe J and El-Osta A: Epigenetic phenomena linked to diabetic complications. Nat Rev Endocrinol 6: 665-675, 2010.

19. Marmorstein R and Trievel RC: Histone modifying enzymes: Structures, mechanisms, and specificities. Biochim Biophys Acta 1789: 58-68, 2009.

20. Shilatifard A: Chromatin modifications by methylation and ubiquitination: Implications in the regulation of gene expression. Annu Rev Biochem 75: 243-269, 2006.

21. Sun G, Reddy MA, Yuan H, Lanting L, Kato M and Natarajan R: Epigenetic histone methylation modulates fibrotic gene expression. J Am Soc Nephrol 21: 2069-2080, 2010.

22. Reed SM and Quelle DE: p53 Acetylation: Regulation and consequences. Cancers (Basel) 7: 30-69, 2014.

23. Marouco D, Garabadgiu AV, Melino G and Barlev NA: Lysine-specific modifications of p53: A matter of life and death? Oncotarget 4: 1556-1571, 2013.

24. Lee JT and Gu W: SIRT1: Regulator of p53 deacetylation. Genes Cancer 4: 112-117, 2013

25. Shi Y, Sawada J, Sui G, Affar el B, Whetstine JR, Lan F, Ogawa H, Luke MP, Nakatani Y and Shi Y: Coordinated histone modifications mediated by a CtBP co-repressor complex. Nature 422: 735-738, 2003

26. Shi Y, Lan F, Matson C, Mulligan P, Whetstine JR, Cole PA, Casero RA and Shi Y: Histone demethylation mediated by the nuclear amine oxidase homolog LSD1. Cell 119: 941-953, 2004.

27. Wang H, Cao R, Xia L, Erdjument-Bromage H, Borchers C, Tempst $\mathrm{P}$ and Zhang Y: Purification and functional characterization of a histone $\mathrm{H} 3$-lysine 4-specific methyltransferase. Mol Cell 8: 1207-1217, 2001.

28. Nishioka K, Chuikov S, Sarma K, Erdjument-Bromage H, Allis CD, Tempst P and Reinberg D: Set9, a novel histone H3 methyltransferase that facilitates transcription by precluding histone tail modifications required for heterochromatin formation. Genes Dev 16: 479-489, 2002. 